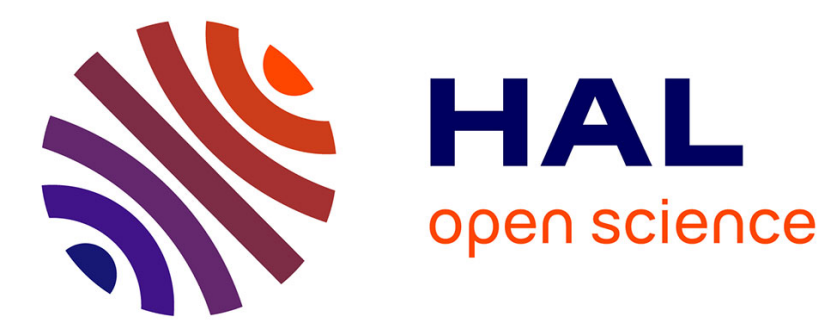

\title{
Polarimetric Incoherent Target Decomposition by Means of Independent Component Analysis
}

Nikola Besic, Gabriel Vasile, Jocelyn Chanussot, Srdjan Stankovic

\section{To cite this version:}

Nikola Besic, Gabriel Vasile, Jocelyn Chanussot, Srdjan Stankovic. Polarimetric Incoherent Target Decomposition by Means of Independent Component Analysis. IEEE Transactions on Geoscience and Remote Sensing, 2015, 53 (3), pp.1236-1247. 10.1109/TGRS.2014.2336381 . hal-01023169

\section{HAL Id: hal-01023169 https://hal.science/hal-01023169}

Submitted on 15 Jul 2014

HAL is a multi-disciplinary open access archive for the deposit and dissemination of scientific research documents, whether they are published or not. The documents may come from teaching and research institutions in France or abroad, or from public or private research centers.
L'archive ouverte pluridisciplinaire $\mathbf{H A L}$, est destinée au dépôt et à la diffusion de documents scientifiques de niveau recherche, publiés ou non, émanant des établissements d'enseignement et de recherche français ou étrangers, des laboratoires publics ou privés. 


\title{
Polarimetric Incoherent Target Decomposition by Means of Independent Component Analysis
}

\author{
Nikola Besic, Student Member IEEE, Gabriel Vasile, Member IEEE, Jocelyn Chanussot, Fellow IEEE, \\ and Srdjan Stankovic, Senior Member IEEE
}

\begin{abstract}
This paper presents an alternative approach for polarimetric incoherent target decomposition dedicated to the analysis of very-high resolution POLSAR images. Given the nonGaussian nature of the heterogeneous POLSAR clutter due to the increase of spatial resolution, the conventional methods based on the eigenvector target decomposition can ensure uncorrelation of the derived backscattering components at most. By introducing the Independent Component Analysis (ICA) in lieu of the eigenvector decomposition, our method is rather deriving statistically independent components. The adopted algorithm - FastICA, uses the non-Gaussianity of the components as the criterion for their independence. Considering the eigenvector decomposition as being analogues to the Principal Component Analysis (PCA), we propose the generalization of the ICTD methods to the level of the Blind Source Separation (BSS) techniques (comprising both PCA and ICA). The proposed method preserves the invariance properties of the conventional ones, appearing to be robust both with respect to the rotation around the line of sight and to the change of the polarization basis. The efficiency of the method is demonstrated comparatively, using POLSAR Ramses $\mathrm{X}$-band and ALOS L-band data sets. The main differences with respect to the conventional methods are mostly found in the behaviour of the second most dominant component, which is not necessarily orthogonal to the first one. The potential of retrieving non-orthogonal mechanisms is moreover demonstrated using synthetic data. On expense of a negligible entropy increase, the proposed method is capable of retrieving the edge diffraction of an elementary trihedral by recognizing dipole as the second component.
\end{abstract}

Index Terms-ICTD, ICA, non-Gaussianity, independence, non-orthogonality

\section{INTRODUCTION}

Target decomposition (TD), introduced in the first place in [1], aims to interpret polarimetric data by assessing and analysing the components involved in the scattering process [2]. When estimating scattering components, conventional algebraic incoherent target decompositions (ICTD) rely on the Hermitian nature of the positive semi-definite target coherence (or covariance) matrix [3], [4]. Eigenvector decomposition of the space averaged coherence matrix results in a set of mutually orthogonal target vectors, representing the three dominant

Manuscript received October 5, 2013; revised April 9, 2014; accepted June 29, 2014.

N. Besic is with the GIPSA-lab, Grenoble INP, Grenoble, France and with the University of Montenegro, Podgorica, Montenegro, (e-mail: nikola.besic@gipsa-lab.grenoble-inp.fr).

G. Vasile is with the GIPSA-lab, CNRS.

J. Chanussot is with the GIPSA-lab, Grenoble INP and with the Faculty of Electrical and Computer Engineering, University of Iceland, Reykjavik, Iceland.

S. Stankovic is with the University of Montenegro. single scatterers in a scene. Accompanying eigenvalues define the contribution of the corresponding scatterers to the total scattering. The product of the derived target vector with its Hermitian conjugate, multiplied by the matching eigenvalue, results in the coherence matrix of a single scatterer. Therefore, using the coherence matrix, the backscattering mechanism is expressed as the incoherent sum of three elementary mechanisms, considered as independent. The two essentially used algebraic decompositions are the $H / \alpha$ decomposition, proposed by Cloude and Pottier [5] and the Touzi decomposition [6]. They differ in terms of parametrization of the derived target vectors: the first one uses $\alpha-\beta-\gamma-\delta$ model, while the second uses the Target Scattering Vector Model (TSVM), which ensures roll-invariance in case of both symmetric and non-symmetric targets.

The Independent Component Analysis (ICA) is a Blind Source Separation technique (BSS) aiming to recover independent source signals without having any physical knowledge of the mixing process [7]. Unlike the Principal Component Analysis (PCA), as well considered a BSS technique, but limited to the second order statistics of the observations [8], the ICA is rather based on the knowledge of higher order statistical moments. The PCA results in statistically uncorrelated sources at best, which appears to be sufficient in case of Gaussian observations where uncorrelated equals to independent [9]. However, if the observed mixture signals, analysed as random variables, are not Gaussian, which happens to be simultaneously the basic condition for applying the ICA, independence turns out to be a more rigorous way of discrimination than uncorrelation [10].

Under certain constraints, the eigenvector decomposition of the scattering coherence matrix, provides the same results as the PCA of the corresponding representative target vector [11]. Thus, the conventional approach in POLSAR images target decomposition results in deriving uncorrelated components. This is adequate if we consider the conventional statistical model assuming Gaussian homogeneous clutter [12]. However, given the improvement in spatial resolution, the POLSAR data can be rather characterised by non-Gaussian heterogeneous clutter [13]. In this case decorrelation cannot be considered as the most meticulous way for separating the scattering sources present in the scene. It appears that more advantageous solutions, capable of deriving independent components, are needed. Applying the ICA seems to be one of such solutions.

The ICA method have been already successfully employed on SAR data: in speckle reduction, feature extraction and data fusion [14], [15]. The application on polarimetric data 
was, however, either restricted on analysis of two-components polarimetric target vector [16], either rather related to the POLInSAR data analysis [17].

The main idea of this article is to propose a generalization of the polarimetric decompositions to the level of blind source separation techniques by introducing the ICA method instead of the eigenvector decomposition. Essentially, our motivation is the possibility to exploit higher order statistics of the nonGaussian target vector in order to recover a set of independent dominant scatterers. In this particular case, the recovered linearly independent scattering target vectors are not necessarily mutually orthogonal, which is demonstrated using a synthetic data set. At first, we apply the statistical classification algorithm (for example [18]) in order to obtain stationary sets of polarimetric observations - scattering matrices projected onto the Pauli basis. Then, the target vectors of the single scatterers are estimated by applying Complex Non-Circular FastICA algorithm [19] on each of the sets derived in the previous step. They are parametrised using the TSVM, allowing the Poincaré sphere representation with direct physical interpretation [20]. The share of the component in the total backscattering is computed by the squared $\ell^{2}$ norm of the single scatterer target vector. The proposed method is invariant, both under the rotation around the line of sight (LOS) and under the change of polarization basis. The latter is demonstrated using the projection of the observations onto the circular polarization basis, coupled with the Circular Polarization Scattering Vector (CPSV) model [21] and furthermore, by additionally employing $\alpha-\beta-\gamma-\delta$ model in Pauli basis [5].

The method particularities with respect to the conventional approach are demonstrated using RAMSES X-band and ALOS L-band data sets. Comparative analysis points out strong similarity when dealing with the first most dominant components. However, there is a remarkable difference in the behaviour of the second components. It appears that on the expense of a negligible increase in entropy, the second most dominant component contains some valuable information. In the first data set, acquired over urban area, while analysing the class which corresponds to the elementary trihedral placed in the scene, we detect the diffraction scattering by identifying dipole as the second most dominant component. On the other side, when dealing with the distributed targets (mountainous region), we are able to rely on the symmetry of the second most dominant component in discriminating between different types of snow cover and the bare ground. In terms of the second component, we demonstrate also the advantage of the "global" (classification) over the "local" approach (sliding window) in selecting observation datasets.

The article is organized as follows: in Chapter II we are discussing the main differences between the existing (PCA) and the proposed solution (ICA), in terms of statistics. Further, chapter III contains the description of the method, comprising the details concerning the applied ICA algorithm and an introduction to the Touzi's TSVM parametrisation [6]. The roll-invariance properties are as well discussed in this chapter. Application on two real data-sets, followed by corresponding discussion are given in chapter IV. This chapter contains an application on a synthetic data set as well, used to demonstrate the capability of retrieving non-orthogonal mechanisms. The polarization basis invariance analysis is demonstrated using one of the real data sets. Finally, chapter V concludes the article and provides future perspectives.

\section{PCA AND ICA}

Blind Source Separation techniques use a set of observation vectors $(\mathrm{x})$ to retrieve the sources vector $(\mathbf{s})$ and the mixing matrix (A), which gives the share of the sources in the observed process [22]:

$$
\begin{aligned}
{\left[\begin{array}{l}
x_{1}(i, j) \\
x_{2}(i, j) \\
x_{3}(i, j)
\end{array}\right] } & =\left[\begin{array}{lll}
A_{11}^{\prime} & A_{12}^{\prime} & A_{13}^{\prime} \\
A_{21}^{\prime} & A_{22}^{\prime} & A_{23}^{\prime} \\
A_{31}^{\prime} & A_{32}^{\prime} & A_{33}^{\prime}
\end{array}\right] \cdot\left[\begin{array}{l}
s_{1}^{\prime}(i, j) \\
s_{2}^{\prime}(i, j) \\
s_{3}^{\prime}(i, j)
\end{array}\right]= \\
& =\left[\begin{array}{lll}
A_{11}^{\prime \prime} & A_{12}^{\prime \prime} & A_{13}^{\prime \prime} \\
A_{21}^{\prime \prime} & A_{22}^{\prime \prime} & A_{23}^{\prime \prime} \\
A_{31}^{\prime \prime} & A_{32}^{\prime \prime} & A_{33}^{\prime \prime}
\end{array}\right] \cdot\left[\begin{array}{l}
s_{1}^{\prime \prime}(i, j) \\
s_{2}^{\prime \prime}(i, j) \\
s_{3}^{\prime \prime}(i, j)
\end{array}\right] .
\end{aligned}
$$

However, the criterion for separation varies upon the method being used. Principally, the sources are expected to be either mutually uncorrelated $\left(\mathbf{s}^{\prime}\right)$ or mutually independent $\left(\mathbf{s}^{\prime \prime}\right)$ (if the higher order statistical moments differ from zero, which is the case for non-Gaussian observations). The former criterion is used in the Principal Component Analysis, while the latter is associated to the Independent Component Analysis.

If we introduce the spatially averaged covariance matrix of the observation vector as $\mathbf{C}_{x}$, the mixing matrices $\mathbf{A}^{\prime}$ and $\mathbf{A}^{\prime \prime}$ can be represented as factorizations of the covariance matrix [11]:

$$
\mathbf{C}_{x}=\mathbf{A}^{\prime} \mathbf{A}^{\prime \dagger}=\mathbf{A}^{\prime \prime} \mathbf{A}^{\prime \prime \dagger}
$$

and they are mutually related by an unitary matrix $\mathbf{P}$ :

$$
\mathbf{A}^{\prime \prime}=\mathbf{A}^{\prime} \mathbf{P} \text {. }
$$

Choosing the columns of $\mathbf{A}^{\prime}$ to be denormalized eigenvectors of $\mathbf{C}_{x}$, ensures decorrelation between the elements of $\left(\mathbf{s}^{\prime}\right)$. The denormalization assumes multiplying by the square root of eigenvalue and it is emphasized since it is the denormalized eigenvector which forms a coherence matrix of a single scatterer in a conventional approach. Even though multiplication of $\mathbf{A}^{\prime}$ with an arbitrary unitary matrix (rotation) preserves decorrelation, maximum energies for the components of $\mathbf{s}^{\prime}$ are achieved with the matrix of eigenvectors. For this reason, we identify the first step of the conventional approach in ICTD (eigenvector decomposition) with the PCA [11].

On the other side, matrix $\mathbf{A}^{\prime \prime}$ cannot be retrieved using only second-order statistics, unless we treat Gaussian observations. Even though it is intrinsically linked to the matrix $\mathbf{C}_{x}$, the mixing matrix of independent sources cannot be estimated using this matrix only. Namely, considering the equation 3, it appears that the estimation of the unitary "floating" matrix $\mathbf{P}$ requires knowledge of the higher order statistics.

Therefore, it is necessary to apply a method capable of exploiting higher order statistical moments - the ICA. The ICTD method, proposed in this article, is based on the fixedpoint FastICA algorithm which relies on non-Gaussianity as a measure of the statistical independence [10], [19]. 


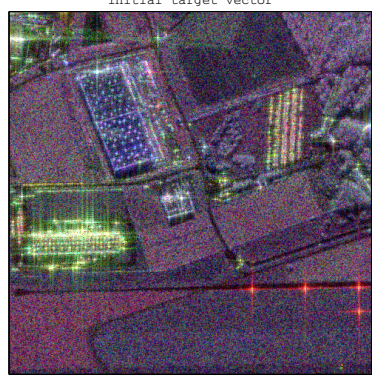

(a)

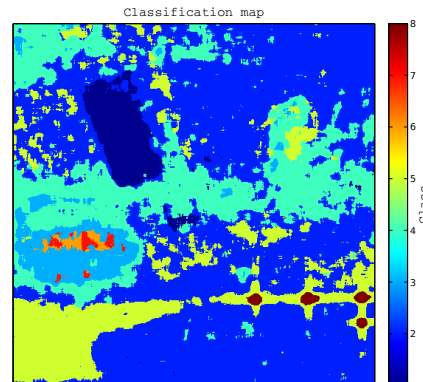

(b)
Fig. 1: RAMSES POLSAR X-band, Brétigny, France: (a) Pauli RGB coded image, (b) Statistical classification performed in the first step.

\section{METHOD}

The proposed incoherent polarimetric decomposition method consists in three main steps:

- Data selection - the observation data sets are selected using statistical classification of the POLSAR image ("global approach"). This choice, rather than a sliding window ("local approach") is discussed in the following section, dealing with the performance analysis.

- Estimation of the independent components - Non-Circular (NC) FastICA algorithm is applied on each of the formerly derived data sets in order to estimate the most dominant single scatterers.

- Parametrization - derived target vectors are parametrized using the Touzi's Target Scattering Vector Model [6].

After the first step, we can assume having defined stationary sets of observed target vectors. The novelty with respect to both the $H / \alpha$ and the Touzi's decompositions is introduced mostly in the second stage.

\section{A. Estimation of the independent components}

The core of the novel ICA based polarimetric decomposition is the estimation of the mixing matrix $\mathbf{A}^{c}$, for each of the derived classes $(c)$. There are several criteria for determining the elements of $\mathbf{A}^{c}$ in order to ensure the mutual independence of the sources in $\mathbf{s}^{c}$. The common factor for all of them is the assumption of high-resolution polarimetric SAR images [23] at most one of the sources is Gaussian and thus their mixture or the observation data prove to be non-Gaussian [10]:

$$
\mathbf{k}^{c}(i, j)=\left[\begin{array}{lll}
A_{11}^{c} & A_{12}^{c} & A_{13}^{c} \\
A_{21}^{c} & A_{22}^{c} & A_{23}^{c} \\
A_{31}^{c} & A_{32}^{c} & A_{33}^{c}
\end{array}\right] \cdot\left[\begin{array}{l}
s_{1}^{c}(i, j) \\
s_{2}^{c}(i, j) \\
s_{3}^{c}(i, j)
\end{array}\right]=\mathbf{A}^{c} \mathbf{s}^{c}(i, j) .
$$

In our case, the observation data are the Pauli target vectors corresponding to the a priori defined class $\left(\mathbf{k}_{c} \in \mathbf{K}_{c}\right)$, meaning that we finally obtain one mixing matrix $\mathbf{A}^{c}$ for each of the classes $c$. In the particular case of ICTD, we are facing the complex nature of the observation data.

By applying different criteria in the Complex FastICA algorithm, we compare, in the framework of ICTD, the performances of several strategies used in the estimation of the complex independent components [24]. The selected approach is specifically adapted to the scenario where sources may eventually exhibit non-circular distributions [19].

Pre-processing of the observation data consists in centering and whitening. The former assumes subtracting the mean values, making the estimated sources inconvenient for taking over the intuitive role of the eigenvalues. The later is the orthogonalization transform $\mathbf{V}$ applied on the set of vectors $\mathbf{k}^{c}$ and therefore on the mixing matrix $\mathbf{A}^{c}$ as well:

$$
\tilde{\mathbf{k}}=\mathbf{E} \mathbf{D}^{-1 / 2} \mathbf{E}^{H} \mathbf{k}^{c}=\mathbf{V} \mathbf{k}^{c}=\mathbf{V} \mathbf{A}^{c} \mathbf{s}^{c}=\tilde{\mathbf{A}} \mathbf{s}^{c},
$$

with $\mathbf{E}$ being a matrix of eigenvectors of $\mathbb{E}\left\{\mathbf{k}^{c} \mathbf{k}^{c H}\right\}$ and D a diagonal matrix containing corresponding eigenvalues. However, at this stage, the components are not scrupulously decorrelated, which can be deduced from non-diagonalized pseudo-covariance matrix $\mathbb{E}\left\{\tilde{\mathbf{k}} \tilde{\mathbf{k}}^{T}\right\}$ [19].

The FastICA algorithm is a fast converging algorithm based on a fixed-point iteration scheme for finding the global nonGaussianity maximum for each estimated source $y=\mathbf{w}^{H} \tilde{\mathbf{k}}$ [25], with $\tilde{\mathbf{k}}$ being the whitened observation data vector and $\mathrm{w}$ the mixing vector (column of the estimated mixing matrix $\mathbf{W})$ converging to one of the columns of the whitened mixing matrix $\tilde{\mathbf{A}}$ ( $y$ converging to the corresponding source $s$ ).

The Complex FastICA algorithm is based on a bottomup approach: emphasizing the non-Gaussanity of the sources by maximizing an arbitrary nonlinear contrast function whose extrema coincides with the independent component [26]:

$$
J_{G}(\mathbf{w})=\mathbb{E}\left\{G\left(\left|\mathbf{w}^{H} \tilde{\mathbf{k}}\right|^{2}\right)\right\} .
$$

The performances of the algorithm strongly depend on the choice of the nonlinear function $G(y)$, which is supposed to be suited to the particular application. Therefore, we use here three different functions, leading to different criteria $(C)$ in deriving the independent target vectors:

- kurtosis (C1):

$$
G_{1}(y)=\frac{1}{2} y^{2}
$$

- logarithm (C2):

$$
G_{2}(y)=\log (0.05+y)
$$

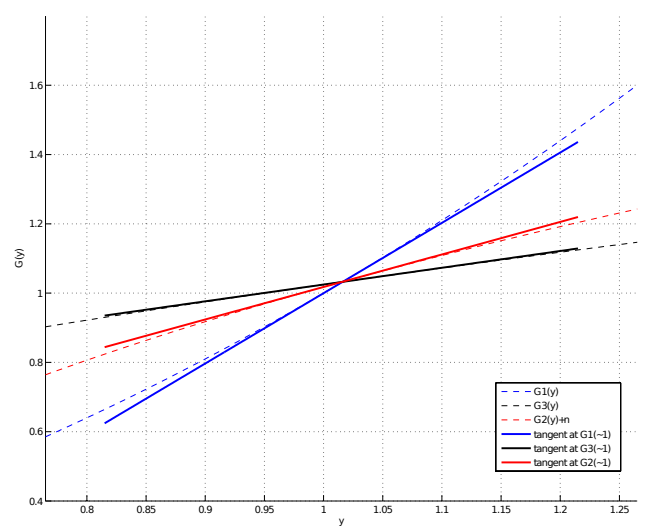

Fig. 2: Nonlinear functions used in the Complex Non-Circular FastICA algorithm, along with the tangents in the point $\sim 1$, indicating the difference in the monotony. 


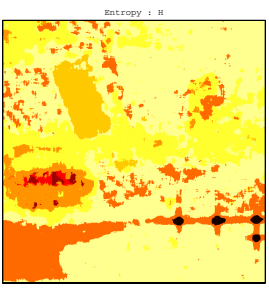

(a)

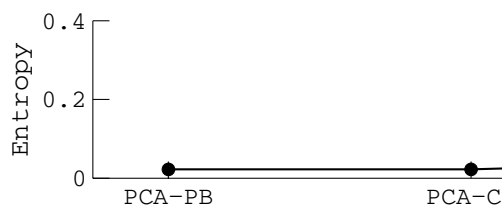

b)

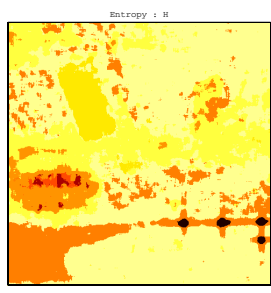

(c)

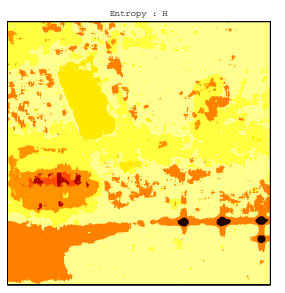

(d)

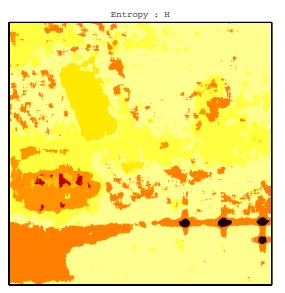

(e)

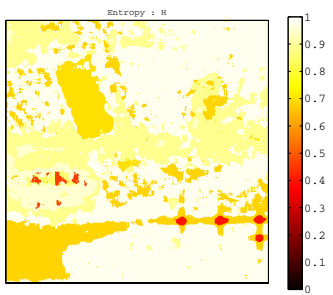

(f)

Fig. 3: RAMSES POLSAR X-band, Brétigny, France: entropy estimation using (a) PCA - Pauli basis, (b) PCA - circular basis, (c) ICA - Pauli basis (C2 criterion), (d) ICA - circular basis (C2 criterion), (e) ICA - Pauli basis (C3 criterion), (f) ICA - Pauli basis (C1 criterion), (g) Entropy estimated for the trihedral class (Class 8).

- square root $(C 3)$ :

$$
G_{3}(y)=\sqrt{0.05+y}
$$

In the first case, the contrast functions becomes essentially a measure of the fourth statistical moment of the source kurtosis. As its value in case of the Gaussian variable equals zero, by maximizing the kurtosis of each of the sources, we ensure their independence. Being slowly growing nonlinear functions (Fig. 2), $G_{2}(y)$ and $G_{3}(y)$ allow more robust estimation with respect to the presence of outliers.

Additionally, by including the pseudo-covariance matrix of the observation target vectors in maximizing the contrast function (Eq. 6), the applied algorithm is generalized to the case of complex sources having a non-circular distribution [19]. This way, despite the modulus in Eq. 6, the phase information is preserved.

Finally, the estimated mixing matrix is de-whitened by using the inverse orthogonalisation transform $\mathbf{V}^{-1}$ :

$$
\mathbf{A}^{c}=\mathbf{V}^{-1} \mathbf{W}
$$

The result of the incoherent target decomposition is the set of target vectors representing elementary scatterers and a set of scalars, providing their proportion in the total scattering. In our case, the target vectors of the independent scatterers are the columns of the estimated de-whitened mixing matrix $\mathbf{A}^{c}$.

The contributions to the total backscattering are computed as the squared $\ell^{2}$ complex norms of the mixing matrix columns - the energies of the single scatterers [27]:

$$
\left\|\mathbf{A}_{\mathbf{i}}\right\|_{2}^{2}=\left|A_{1 i}\right|^{2}+\left|A_{2 i}\right|^{2}+\left|A_{3 i}\right|^{2} .
$$

In the framework of the formalism introduced in the section II, the contributions summed up in Eq. 11 could be defined as diagonal elements of the matrix $\mathbf{A}^{\prime \prime} \mathbf{A}^{\prime \prime \dagger}$. In the same way, eigenvalues are diagonal elements of $\mathbf{A}^{\prime} \mathbf{A}^{\prime \dagger}$. Even though the matrix $\mathbf{A}^{\prime \prime}$ is not orthogonal and therefore some information contained in the non-diagonal elements of $\mathbf{A}^{\prime \prime} \mathbf{A}^{\prime \prime \dagger}$ is lost, the entropy estimated in these two cases is significantly similar. In the earlier attempt of introducing the ICA into the POLSAR data analysis [17], the contributions were estimated by rather relying on the derived sources $\left(P_{i}=\frac{1}{3} \mathbf{s}(i) \mathbf{s}^{H}(i)\right)$, which doesn't appear to be an appropriate choice, given the variances of the estimated sources being set to the unit value.

\section{B. Parametrization}

Being based on Kennaugh-Huynen condiagonalization [28], [2] projected onto the Pauli basis, the TSVM [6] allows the parametrization of the target vector in terms of rotation angle $(\psi)$, phase $\left(\Phi_{s}\right)$, maximum amplitude $(m)$, target helicity $\left(\tau_{m}\right)$, symmetric scattering type magnitude $\left(\alpha_{s}\right)$ and symmetric scattering type phase $\left(\Phi_{\alpha_{s}}\right)$, among which the last four are roll-invariant:

$$
\mathbf{k}=m|\mathbf{k}|_{m} e^{j \Phi_{s}}\left[\begin{array}{ccc}
1 & 0 & 0 \\
0 & \cos 2 \psi & -\sin 2 \psi \\
0 & \sin 2 \psi & \cos 2 \psi
\end{array}\right]\left[\begin{array}{c}
\cos \alpha_{s} \cos 2 \tau_{m} \\
\sin \alpha_{s} e^{j \Phi_{\alpha_{s}}} \\
-j \cos \alpha_{s} \sin 2 \tau_{m}
\end{array}\right] .
$$

In order to avoid an ambiguity related to the KennaughHuynen condiagonalization, the range of the orientation angle is reduced to the $[-\pi / 4, \pi / 4]$, by introducing the identity:

$\mathbf{k}\left(\Phi_{s}, \psi, \tau_{m}, m, \alpha_{s}, \Phi_{\alpha_{s}}\right)=\mathbf{k}\left(-\Phi_{s}, \psi \pm \pi / 2,-\tau_{m}, m, \alpha_{s},-\Phi_{\alpha_{s}}\right)$.

Using TSVM parameters, it is eventually possible to represent the obtained independent target vectors on either symmetric or non-symmetric target Poincaré sphere [29], [30]. In our case, they do not necessarily form an orthogonal basis.

\section{Roll-Invariance}

One of the major conveniences of the conventional approach is the roll-invariance of the coherence matrix constructed from a linear combination of the eigenvectors [3]. Even though the proposed method does not directly conserve the roll-invariance through the reconstruction from a linear combination of the eigenvectors, it appears as well to be invariant to the rotation $\mathbf{R}(\theta)$ of the observed target vectors (Table III): 


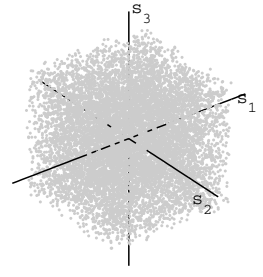

(a)

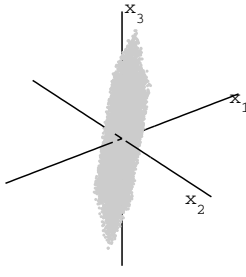

(b)

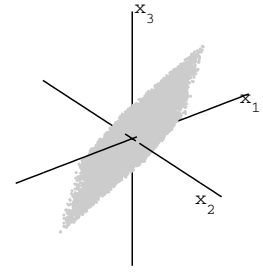

(c)
Fig. 4: The joint probability density functions of: (a) three uniformly distributed real sources, (b) the mixture, (c) the mixture rotated about the line of sight ( $x_{1}$ axis) for $\theta=30^{\circ}$.

$$
\mathbf{k}^{c \theta}=\mathbf{R}(\theta) \mathbf{k}^{c}=\left[\begin{array}{ccc}
1 & 0 & 0 \\
0 & \cos (2 \theta) & -\sin (2 \theta) \\
0 & \sin (2 \theta) & \cos (2 \theta)
\end{array}\right] \mathbf{k}^{c} .
$$

In order to prove and justify this, we ought to reconsider the formerly introduced ICA method in a rather "geometrical" manner. For instance, let us presume having three uniformly distributed real sources $\mathbf{s}$ (Fig. 4a). By multiplying them by a non-orthogonal mixing matrix $\mathbf{A}$, we get the set of observations $\times$ (Fig. $4 b$ ). The role of the ICA algorithm is to find a mixing matrix which does exactly the inverse transforms the data from the space in Fig. $4 \mathrm{~b}$ to the one given in Fig. 4a. Given that the whitening and de-whitening deal with the form (transforming data in Fig. 4b into "the cube"), the estimated mixing matrix actually accounts for the orientation of "the cube", representing a 3D rotation, defined with three angles corresponding to three degrees of freedom.

Switching to the complex domain does not change the essence of the presented "geometrical" interpretation. In the context of our application $\left(x=\mathbf{k}^{c}\right)$, with implicitly assumed whitening and de-whitening, the rotation around the line of sight $\mathbf{R}(\theta)$ affects only one of the angles defining $\mathbf{A}(\phi, \xi, \chi)$ (Fig. 4c):

$$
\mathbf{k}_{r}^{c}=\mathbf{R}(\theta) \mathbf{k}^{\mathbf{c}}=\mathbf{R}(\theta) \mathbf{A}(\psi, \xi, \chi) \mathbf{s}=\mathbf{A}_{r}(\psi+\theta, \xi, \chi) \mathbf{s}
$$

The other rotation angles $(\xi$ and $\chi)$ remain the same, which do not change with the performed inversions (demixing matrix B) and they provide us the roll-invariant parameters in the estimated mixing matrix $\underline{\mathbf{A}_{r}}$ :

$$
\begin{gathered}
\mathbf{B}=\mathbf{A}_{r}^{-1}=(\mathbf{R A})^{-1}=\mathbf{A}^{-1} \mathbf{R}^{-1}, \\
\underline{\mathbf{A}_{r}}=\mathbf{B}^{-1}=\mathbf{R} \underline{\mathbf{A}} .
\end{gathered}
$$

The columns of the estimated mixing matrix $\mathbf{A}_{r}$ are the estimated backscattering components and, as the derived TSVM parameters are invariant with respect to change of the $\psi$ of the component, they are equally invariant with respect to the change introduced by the rotation applied on the observation target vectors $\psi+\theta$. Therefore, even though the FastICA algorithm itself is not invariant under the rotations of the observation data $\left(\mathbf{A}_{r} \neq \underline{\mathbf{A}}\right)$, the TSVM parameters derived in our case are indeed invariant.
For the purpose of comparison, in this case we neglect the identity in Eq. 13, which however, does not compromise the validity of the derived conclusions.

\section{PERFORMANCE ANALYSIS}

After discussing the data selection criteria, we demonstrate the particularities of the proposed method through the application on two real POLSAR data sets: RAMSES X-band image acquired over Brétigny, France and ALOS L-band images acquired over Chamonix, Mont Blanc, France. Aside from that, using a synthetically generated data set, we emphasise the difference with respect to the conventional approach (PCA).

\section{A. Data selection}

The principal drawback of the proposed method is the size of the observation dataset, which has to be somewhat larger than the size of the sliding window used in the well established methods. The inevitable consequence is the bigger number of the independent components out of which not more that the most dominant three can be estimated [17]. In view of this, rather than using a very large sliding window, we rather rely on a classification algorithm in the data selection.

Therefore, the first step is the classification of the POLSAR image. In this article, we choose to classify the input image using the statistical classifier developed for highly textured POLSAR data [18]. Unlike the classical $H / \alpha / A$ unsupervised classification [3], assuming Gaussian homogeneous clutter and therefore relying on the Sample Covariance Matrix (SCM) estimate, classical mean and Wishart distance [31], [32], the non-Gaussian heterogeneous clutter is taken into account.

Under the Spherically Invariant Random Vector (SIRV) model assumption of the POLSAR clutter [33], [13], the initialization is performed through the $H / \alpha$ unsupervised classification based on the Fixed Point (FP) Covariance Matrix estimator [34]. The barycenters of the initialized classes are calculated iteratively using the Riemannian metric corresponding to the geometric mean [18]. At the end, pixels are assigned using the Wishart criterion.

At this point, we obtain the set of representative target vectors for each of the classes. These vector sets represent the observation data for the BSS, while the selection method assures relevance in the case of incoherent targets.

\section{B. Synthetic data set}

In this section, we demonstrate the capability of retrieving non-orthogonal mechanisms using synthetic POLSAR data [11]. The observation data set is created using the nonorthogonal complex mixing matrix:

$$
\mathbf{A}=\left[\begin{array}{ccccccc}
-0.484 & -0.410 i & 0.051 & +0.202 i & 0.156 & -0.265 i \\
0.055 & -0.304 i & -0.016 & +0.218 i & 0.055 & - & 0.347 i \\
0.005 & + & 0.002 i & 0.617 & -0.150 i & 0.468 & + \\
0.260 i
\end{array}\right]
$$

and three independent sources characterized by the Gamma distribution, each of them having different $k$ and $\theta$ parameters:

$$
\mathcal{G}(s \mid k, \theta)=\frac{1}{\theta^{k} \Gamma(k)} s^{k-1} e^{-\frac{s}{\theta}} .
$$




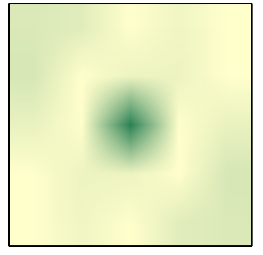

(a)

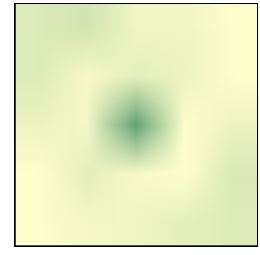

(b)

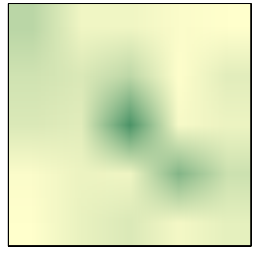

(c)
Fig. 5: Synthetic data set analysis: (a) matrix $A$ autocorrelation, (b) cross-correlation of $A$ and $A^{I C A}$, (c) cross-correlation of $A$ and $A^{P C A}$

The components retrieved in case of applying the ICA algorithm correspond approximately to the components in Eq. 18. On the other side, because the mixing matrix $\mathbf{A}$ is not orthogonal, the PCA is not capable of retrieving the original matrix:

$\mathbf{A}^{I C A}=\left[\begin{array}{ccccccc}-0.446 & -0.402 i & -0.018 & +0.195 i & -0.172 & -0.229 i \\ 0.059 & -0.285 i & -0.092+0.195 i & -0.275 & -0.170 i \\ 0.007 & + & 0.022 i & 0.599+0.119 i & 0.395 & -0.306 i\end{array}\right]$,

$\mathbf{A}^{P C A}=\left[\begin{array}{ccccc}-0.463+0.223 i & 0.562+0.507 i & -0.267-0.303 i \\ 0.839+0.157 i & 0.044+0.437 i-0.107-0.258 i \\ 0.085 & 0.483 & 0.871\end{array}\right]$.

This is confirmed through the analysis of their 2D crosscorrelations [35] illustrated in Fig. 5:

$$
\mathbf{C}_{\mathbf{M}_{\mathbf{1}}, \mathbf{M}_{\mathbf{2}}}(k, l)=\sum_{m=0}^{M-1} \sum_{n=0}^{N-1} \mathbf{M}_{\mathbf{1}}(m, n) \mathbf{M}_{\mathbf{2}}{ }^{\dagger}(m-k, n-l) .
$$

This section demonstrated that the ICA, aside from being able to assure the independence of the components, identifies the second component without any constrain of orthogonality.

\section{Data set I: Urban area}

The results presented in this section are obtained by applying the proposed ICTD on the RAMSES POLSAR X-band image acquired over Brétigny, France. Fig. 1 illustrates the Pauli RGB coded image and shows the classification map used to define the observation data sets for the ICA algorithm.

1) The criterion selection: The goal is to compare and choose the appropriate Non-Circular FastICA criterion in the context of ICTD (Eq. 7, 8 and 9).

The first point of comparison between the proposed criteria in complex independent components derivation $(C 1, C 2$ and C3) is the possibility of identifying the class of trihedral reflectors present in the scene (Class 8 in Fig. 1). The mask derived from the classification map allows us to select the observation data set containing only target vectors from the regions in the image where the reflectors were placed. Further, one mixing matrix is estimated using each of the three criteria. In each case, the first and the second dominant components are presented on the symmetric scattering target Poincaré sphere (Fig. 6) [6]. The third component parameters are provided in the Table I but, due to the values of helicity and symmetric scattering type phase, the illustration using a sphere is not possible.
The method is able to identify the class corresponding to the trihedral reflectors placed in the scene. A curious fact is that the second dominant component in this case appears to be symmetric as well. The kurtosis criterion results however in both first and second components almost matching trihedral. This indicates apparent "splitting" of the trihedral on the two dominant components, which cannot be granted as a good estimation. On the other side, in case of the logarithm and the square root criteria, the second component, although symmetric, rather represents weaker dipole backscattering.

The second point of comparison is entropy estimation [3] (Fig. 3). Having PCA based classic decomposition as a reference, we compare the overall estimation of entropy (all classes), paying particular attention to the trihedral class. The entropy estimation scheme appears to be far better with the criteria (C2 and $C 3)$.

The overall performance of the analysed ICA criteria in the frame of ICTD, seems to depend directly on the growth rate of the employed nonlinear function. The ICA based on slowly growing nonlinear functions (logarithm and square root) are more efficient in both identifying trihedral as the most dominant backscattering mechanism and, although it is an implication, in estimating entropy.

After choosing the second criterion (C2) as the most appropriate one, we compare the ICA based ICTD with the PCA classic counterpart. The estimated first dominant component is nearly equivalent in both cases (Fig. 7). It was this fact which inclined us toward the comparison of the estimated entropy as one of the criteria for selecting the appropriate non-linearity.

The second component, however, appears to be significantly different (Fig. 7). This is both due to the constraint of mutual orthogonality present in the conventional approach and due to the useful information contained in the higher order statistical moments. The same class used in comparing the different criteria (Class 8) happens to be favourable for demonstrating the utility of the second dominant component (Table I and Fig. 6). Namely, dipole as the second strongest single scatterer indicates the capability of recognizing the trihedral's edge diffraction, eventually.

2) Polarisation basis invariance: The same dataset is used to demonstrate the invariance with respect to more complex unitary transform - the change of the polarization basis. The

TABLE I: RAMSES X-BAND POLSAR DATA OVER BRÉTIGNY, FRANCE: ROLL-INVARIANT PARAMETERS OF THE SINGLE SCATTERERS IN THE TRIHEDRAL CLASS (CLASS 8).

\begin{tabular}{|c|c|c|c|c|}
\hline & Comp. & $\tau_{m}[0]$ & $\alpha_{s}[0]$ & $\Phi_{\alpha_{s}} l^{0}$ \\
\hline & trihedral & 0 & 0 & {$[-90,+90]$} \\
\hline \multirow{3}{*}{ PCA } & $1 \mathrm{st}$ & -0.23 & 0.50 & -51.25 \\
\hline & 2nd & -37.15 & 89.21 & -18.64 \\
\hline & $3 \mathrm{rd}$ & 36.15 & 87.90 & 68.86 \\
\hline \multirow{3}{*}{ ICA (C2) } & $1 \mathrm{st}$ & -0.28 & 0.53 & -27.42 \\
\hline & 2nd & -0.24 & 39.91 & 2.56 \\
\hline & $3 r d$ & 19.84 & 58.49 & 77.92 \\
\hline \multirow{3}{*}{ ICA (C1) } & $1 \mathrm{st}$ & -0.33 & 1.49 & 7.54 \\
\hline & 2nd & -0.42 & 7.34 & -9.60 \\
\hline & $3 \mathrm{rd}$ & 7.11 & 24.82 & -83.22 \\
\hline \multirow{3}{*}{ ICA (C3) } & $1 \mathrm{st}$ & -0.28 & 0.53 & -27.70 \\
\hline & 2nd & -0.36 & 41.20 & -3.33 \\
\hline & $3 r d$ & 5.77 & 54.97 & -68.60 \\
\hline
\end{tabular}




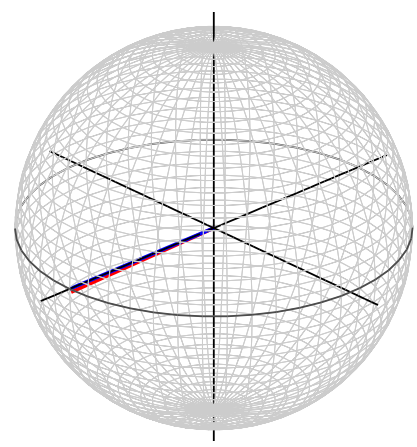

(a)

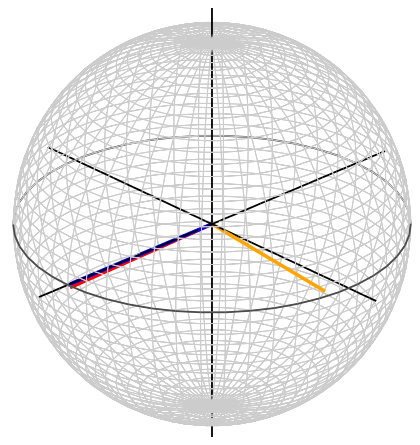

(b)

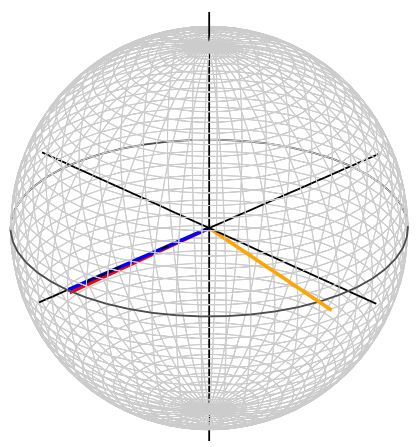

(c)

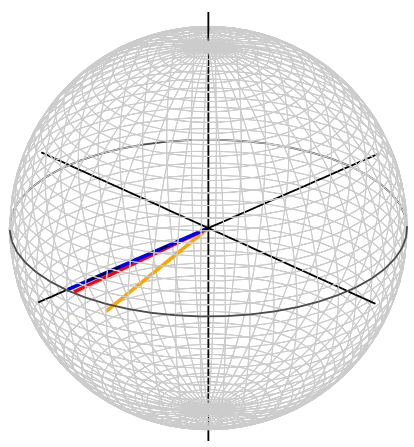

(d)

Fig. 6: RAMSES POLSAR X-band, Brétigny, France: Poincare sphere representation of the trihedral class (Class 8) single scatteres (red - first component, orange - second component, blue - trihedral) using (a) PCA, (b) ICA - C2 criterion, (c) ICA - C3 criterion, (d) ICA - C1 criterion.

observed scattering matrices are projected on the circular polarization basis and the obtained components parametrized using Circular Polarization Scattering Vector (CPSV) [36], [37]:

$$
\begin{aligned}
\mathbf{k}_{c} & =\frac{1}{2}\left[\begin{array}{c}
S_{h h}-S_{v v}+2 j S_{h v} \\
\sqrt{2} j\left(S_{h h}+S_{v v}\right) \\
-S_{h h}+S_{v v}+2 j S_{h v}
\end{array}\right]= \\
& =\sqrt{\operatorname{SPAN}} e^{j \Phi}\left[\begin{array}{c}
\sin \alpha_{c} \cos \beta_{c} e^{j\left(-\frac{4}{3} \Upsilon_{c}-2 \psi\right)} \\
\cos \alpha_{c} e^{j \frac{8}{3} \Upsilon} \\
-\sin \alpha_{c} \sin \beta_{c} e^{j\left(-\frac{4}{3} \Upsilon_{c}+2 \psi\right)}
\end{array}\right] .
\end{aligned}
$$

Among four parameters invariant to the rotation around the LOS $(\psi)$ and to the target absolute phase $(\Phi)$ : energy (SPAN), angle $\Upsilon_{c}$, angle $\alpha_{c}$ and helicity defined as $\mathrm{Hel}_{c}=$ $\sin ^{2} \alpha_{c}\left[\cos ^{2} \beta_{c}-\sin ^{2} \beta_{c}\right]$, we compare the last three with their counterparts derived from TSVM parametrisation in the Pauli basis. The angles $\Upsilon_{c}$ and $\alpha_{c}$, if the target is symmetric $\left(\tau_{m}=0\right)$, correspond, respectively, to $\Upsilon_{\mathrm{TVSM}}=(\pi / 2-$ $\left.\Phi_{\alpha_{s}}\right) / 4$ and $\alpha_{s}$. Helicity $\mathrm{Hel}_{\mathrm{TVSM}}$ is defined as a function of $\tau_{m}$ and the Huynen con-eigenvalues polarizability $\gamma_{H}$ [28], [2]:

$$
\mathrm{Hel}_{\mathrm{TVSM}}=\frac{\cos 2 \gamma_{H} \sin 2 \tau_{m}}{\cos ^{4} \gamma_{H}\left(1+\tan ^{4} \gamma_{H}\right)} .
$$

On one side, as it is demonstrated in the Fig. 8 and in the Table III, we obtain the perfect matching in terms of Hel (if we ignore Eq. 13). On the other side, even for the symmetric classes $\left(\tau_{m} \approx 0\right)$, we don't have a perfect matching of $\Upsilon$, which is justified by the values of $\alpha_{c}$, which converge either to 0 or $\pi / 2$, when this parameters becomes meaningless [21]. The angle $\alpha_{c}$ agrees perfectly with $\alpha_{s}$ in case of symmetric

TABLE II: ALOS L-BAND POLSAR DATA OVER CHAMONIX, MONT BLANC, FRANCE: COMPARISON OF TSVM PARAMETERS OF THE 2ND DOMINANT COMPONENT, OBTAINED EITHER BY MEANS OF PCA OR ICA, FOR THE LABELLED CLASSES.

\begin{tabular}{|c|c|c|c|c|c|c|c|}
\hline Image - & Class & \multicolumn{2}{|c|}{$\tau_{m}\left[^{\circ}\right]$} & \multicolumn{2}{c|}{$\alpha_{s}\left[{ }^{\circ}\right]$} & \multicolumn{2}{c|}{$\Phi_{\alpha_{s}}\left[{ }^{\circ}\right]$} \\
\cline { 3 - 8 } Class & descrip. & PCA & ICA & PCA & ICA & PCA & ICA \\
\hline I-1 & bare gr. & 6.41 & $\mathbf{- 2 . 4 2}$ & 12.64 & 20.33 & 18.45 & -58.20 \\
\hline I-2 & wet snow & -5.83 & $\mathbf{- 1 4 . 8 3}$ & 68.77 & 72.50 & -22.73 & 8.87 \\
\hline II-1 & wet snow & 5.84 & $\mathbf{- 7 . 7 2}$ & 5.55 & 3.45 & 25.96 & -48.09 \\
\hline II-2 & dry snow & -6.96 & $\mathbf{6 . 5 9}$ & 20.22 & 18.49 & -32.46 & 12.17 \\
\hline II-3 & dry snow & -6.47 & $\mathbf{9 . 3 0}$ & 10.27 & 5.50 & -85.02 & -62.80 \\
\hline
\end{tabular}

target. However, in order to reinforce this robustness proof, we introduce $\alpha-\beta-\gamma-\delta$ parametrization, as well [5]:

$$
\begin{aligned}
\mathbf{k}_{p} & =\frac{1}{\sqrt{2}}\left[\begin{array}{c}
S_{h h}+S_{v v} \\
S_{h h}-S_{v v} \\
2 S_{h v}
\end{array}\right]= \\
& =\left|\mathbf{k}_{p}\right| e^{j \theta}\left[\begin{array}{ccc}
1 & 0 & 0 \\
0 & \cos 2 \psi & -\sin 2 \psi \\
0 & \sin 2 \psi & \cos 2 \psi
\end{array}\right]\left[\begin{array}{c}
\cos \alpha_{p} \\
\sin \alpha_{p} \cos \beta_{p} e^{j \delta} \\
\sin \alpha_{p} \sin \beta_{p} e^{j \gamma}
\end{array}\right] .
\end{aligned}
$$

As it can be seen in the Table III, the derived $\alpha_{p}$ parameter, as expected, matches perfectly $\alpha_{c}$, regardless of symmetry. Aside from this, we compare the $\alpha-\beta-\gamma-\delta$ parameters derived conventionally (using PCA) with the ones obtained using our approach. It is the angle $\alpha_{c}$ which fortify the conclusion arising from the TSVM parameters - the first dominant components are quite similar, but the second (non-orthogonal in our case) contains undoubtedly different information.

\section{Data set II: Mountainous region}

In order to analyse the performances in case of a distributed target, the proposed ICTD is applied on POLSAR images acquired over mountainous regions. Two ALOS L-band images of Chamonix, Mont Blanc in France, are used for this purpose. Their classification is given in Fig. 9.

Based on a priori known ground truth ${ }^{1}$, we label the classes (Table II) in two images with one of the four labels (dry snow, wet snow, bare ground and foldover) [20]. Using both the PCA based method (the first and the second component) and the first component of the ICA based method, we do not manage to characterize the labelled classes with any of the derived rollinvariant parameters from Eq. 12. However, the second most dominant component of the ICA based ICTD proves to be useful. As it is demonstrated in Table II, the bare ground can be characterized with helicity parameter close to zero (symmetric target), the dry snow appears to have positive helicity, while the negative values can be associated to the wet snow.

The same data set serves to demonstrate the advantage of the "global approach" (observation data selected using classification) with respect to the "local" one (sliding window based selection). As it can be seen in Figure 9, the local

\footnotetext{
${ }^{1}$ Meteorological data provided by the EDF company.
} 


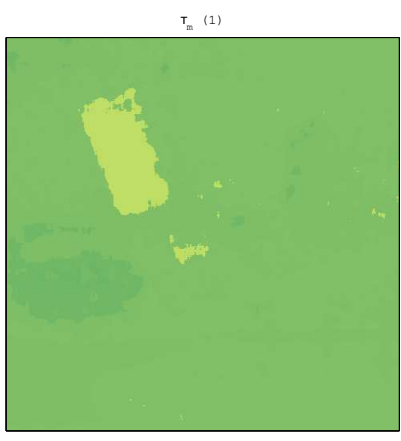

(a)(i)

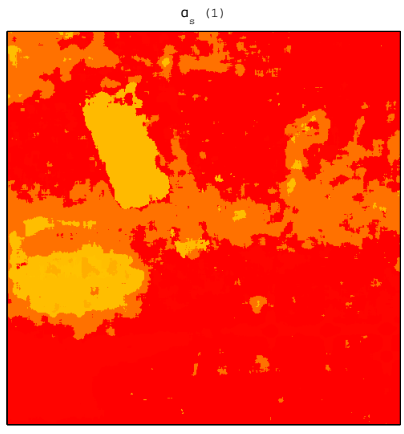

(a)(ii)

$\Phi_{0}(1)$

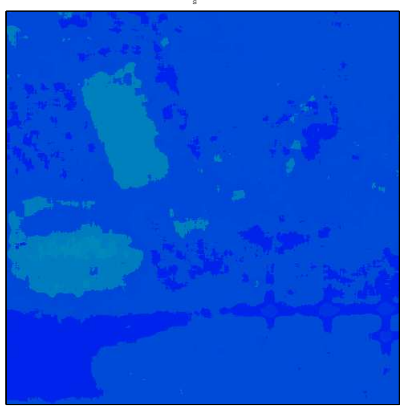

(a)(iii)

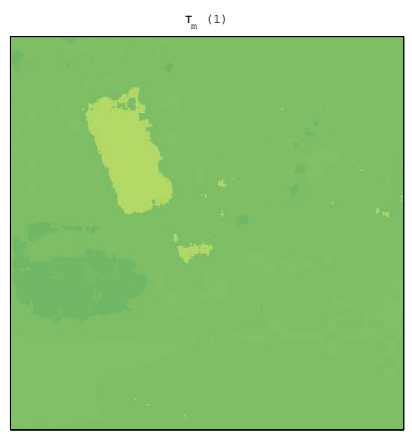

(b)(i)

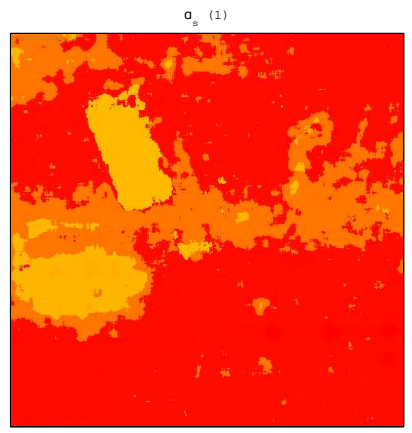

(b)(ii)

$\Phi_{0}(1)$

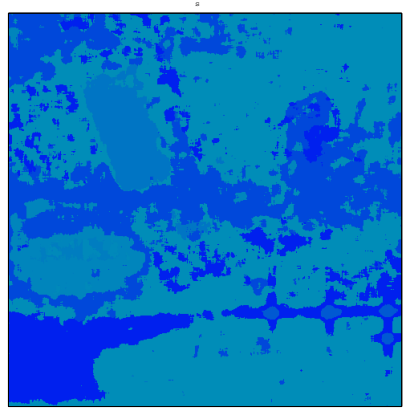

(b)(iii)

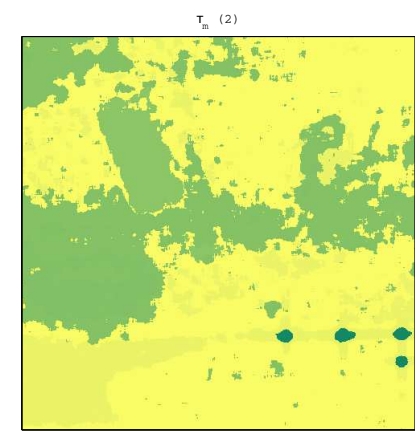

(c)(i)

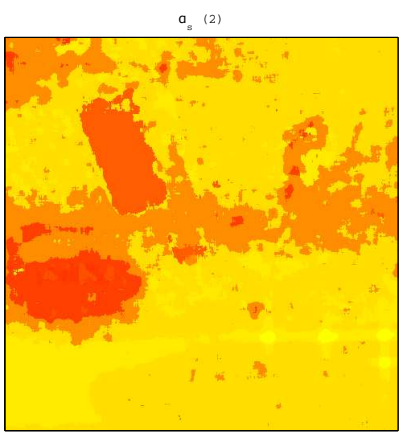

(c)(ii)

$\Phi_{0}(2)$

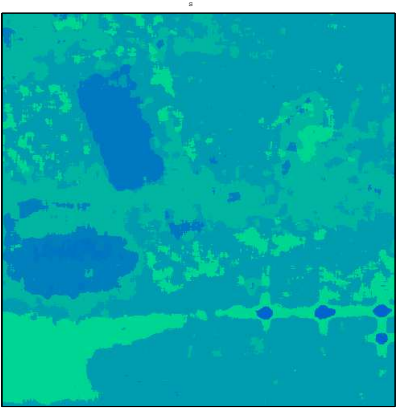

(c)(iii)

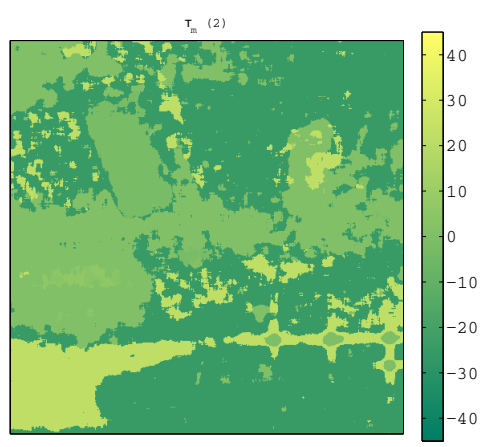

(d)(i)

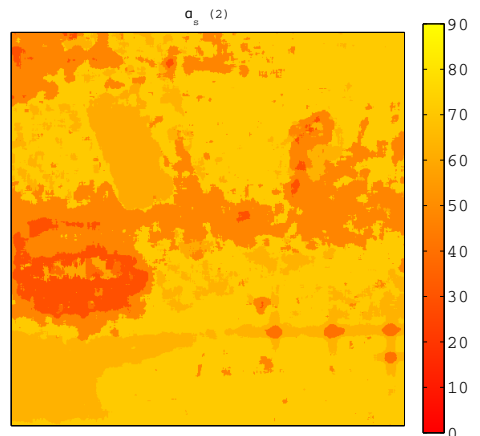

(d)(ii)

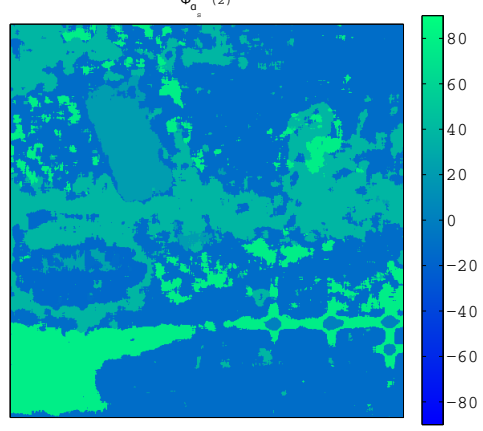

(d)(iii)

Fig. 7: RAMSES POLSAR X-band, Brétigny, France: comparison between the TSVM parameters obtained by means of PCA (first most dominant component (a) and second most dominant component (c)) and by means of ICA (first most dominant component (b) and second most dominant component (d)): (i) $\tau_{m}$, (ii) $\alpha_{s}$, (iii) $\Phi_{\alpha_{s}}$.

approach cannot be used to discriminate between the labelled classes. The reason is the insufficient size of the observation dataset, selected by a sliding window.

\section{CONCLUSiON}

In this article we presented a novel method for Polarimetric Incoherent Target Decomposition, based on the Independent Component Analysis [7]. Motivated by the non-Gaussian nature of the clutter in high resolution POLSAR images, we aimed to exploit higher order statistical moments in retrieving single scatterers present in a scene. Given the stated equivalence between the Principal Component Analysis and the eigenvector decomposition (conventional approach), we generalized ICTD to the level of Blind Source Separation techniques (which comprise both PCA and ICA). After comparing several criteria, the Non-Circular FastICA algorithm [19] based on maximizing the logarithmic non-linear function in order to achieve mutual independence of sources, proved to be the best approach in the framework of ICTD.

The proposed method is able to retrieve non-orthogonal single scatterers, which was illustrated using a synthetic POLSAR data set. It is invariant both under the rotations of the observed target vectors and to the change of the polarization basis.

The results obtained by applying the proposed method on the airborne POLSAR image, acquired over Brétigny, anticipate the potential of the additional information provided by the second dominant component. This was possible by properly taking advantage of both the non-orthogonality property and the higher order statistical moments.

Finally, the second dominant component appeared to be of great interest, also when dealing with the distributed targets. This was illustrated using Touzi's roll-invariant parameters, by achieving a better discrimination between the a priori labelled classes in mountainous regions. 


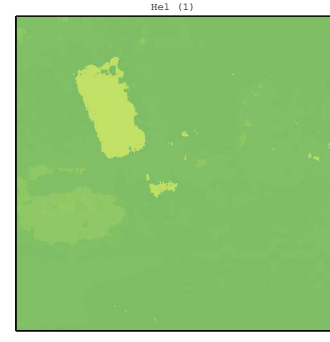

(a)(i)

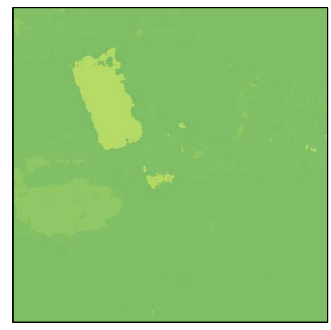

(a)(ii)

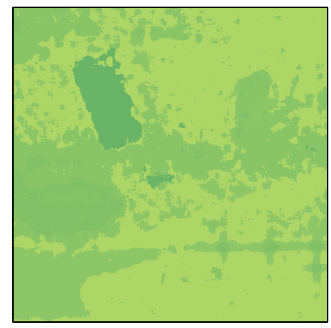

(a)(iii)

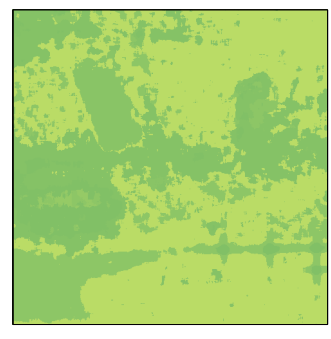

(a)(iv)

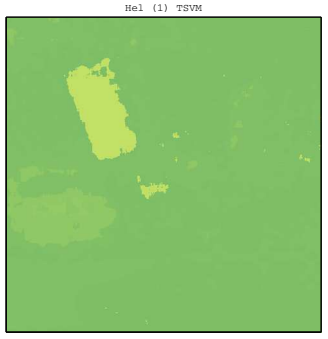

(b)(i)

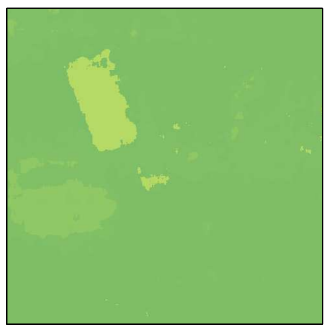

(b)(ii)

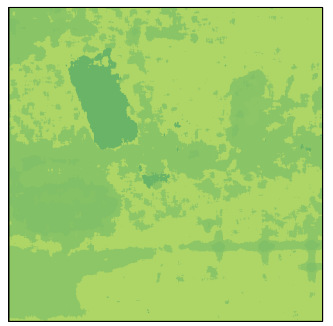

(b)(iii)

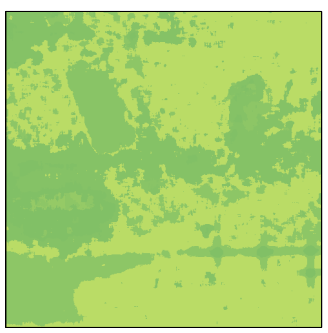

(b)(iv)

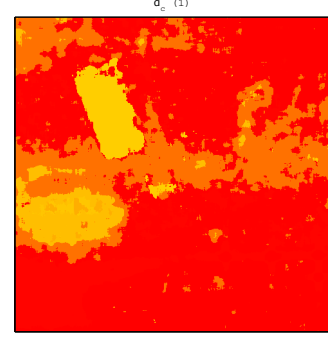

(c)(i)

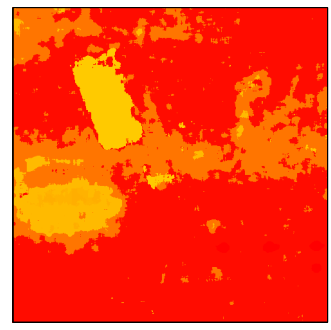

(c)(ii)

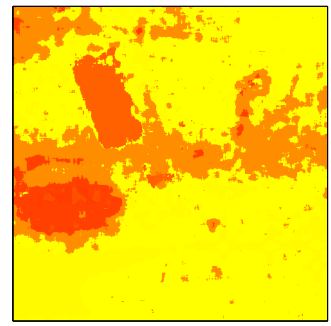

(c)(iii)

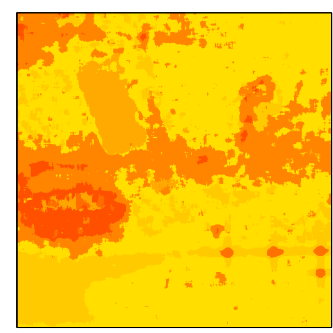

(c)(iv)

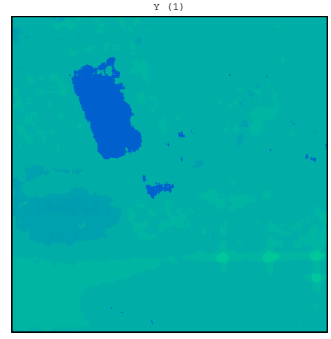

(d)(i)

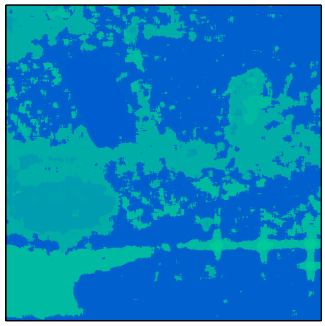

(d),(ii)

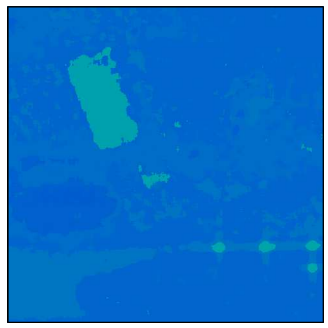

(d)(iii)

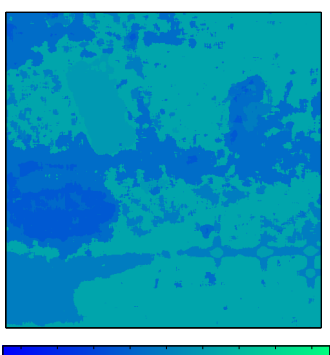

(d)(iv)

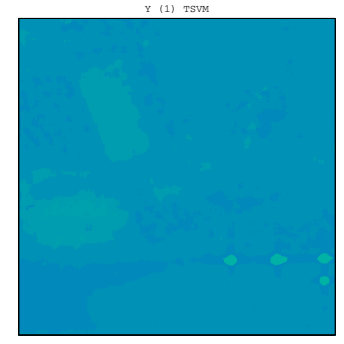

(e)(i)

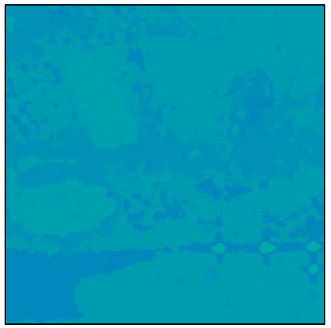

(e),(ii)

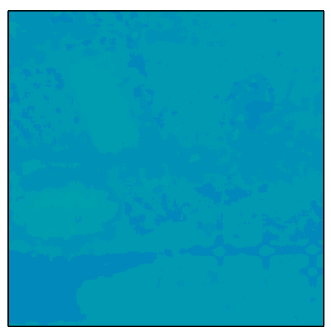

(e)(iii)

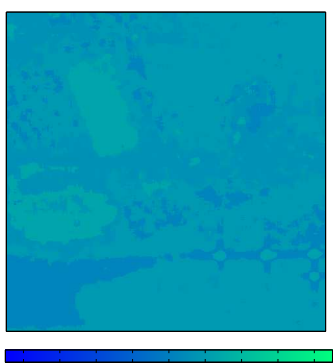

(e)(iv)

Fig. 8: RAMSES POLSAR X-band, Brétigny, France: comparison between the CPSV parameters obtained by means of PCA (first most dominant component (i) and second most dominant component (iii)) and by means of ICA (first most dominant component (ii) and second most dominant component (iv)): (a) $\mathrm{Hel}_{c}$, (b) $\mathrm{Hel}_{\mathrm{TSVM}}$, (c) $\alpha_{c}$, (d) $\Upsilon_{c}$, (e) $\Upsilon_{\mathrm{TSVM}}$.

Future work will enroll in two main directions. Firstly, we will try to explore as much as possible all the benefits of the new information contained in the second dominant component. Secondly, we will continue with applying and comparing different ICA methods in order to achieve the optimal decomposition with respect to the class of stochastic processes under study. The approach which seems to be particularly interesting is to achieve BSS using Maximum Likelihood Estimation.

\section{ACKNOWLEDGEMENT}

The authors would like to thank to the European Space Agency (ESA, AO no. 3638) and the French Aerospace Lab
(ONERA) for providing the POLSAR data sets used in the article. As well, we ought to express gratitude to Électricité de France $(E D F)$ and the Mété-France for providing the ground truth data and other input parameters of interest in case of the snow distributed target. We own gratitude to Dr. Ridha Touzi (Canada Centre for Remote Sensing) for valuable advices regarding the potential role of the second component. The last, but not the least, we would like to thank to anonymous reviewers for their valuable and appreciated advices and comments.

\section{REFERENCES}

[1] J. R. Huynen, "Phenomenological theory of radar targets," Ph.D. dissertation, Technical University, Delft, The Netherlands, 1970. 


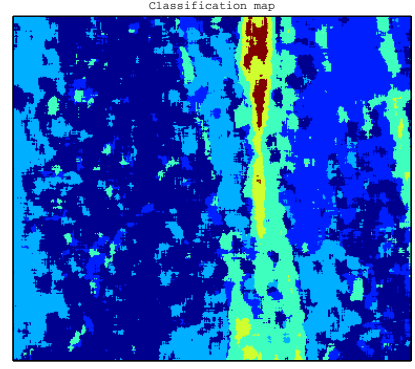

(a)(i)

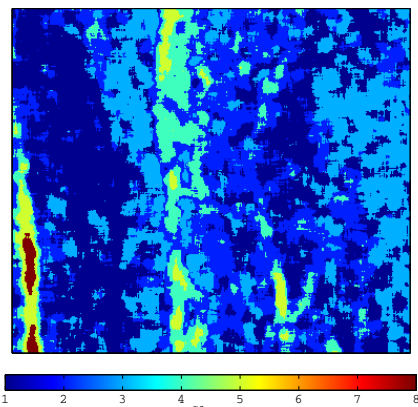

(a)(ii)

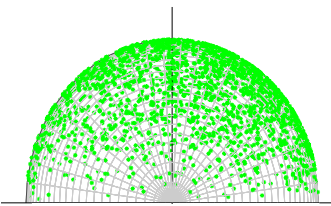

(a)(iii)

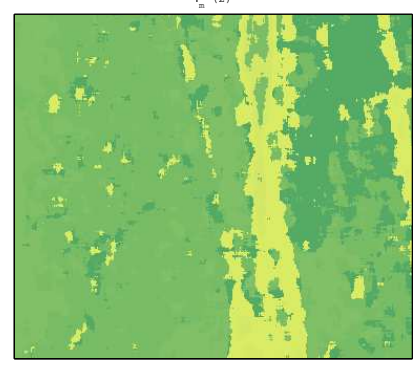

(b)(i)

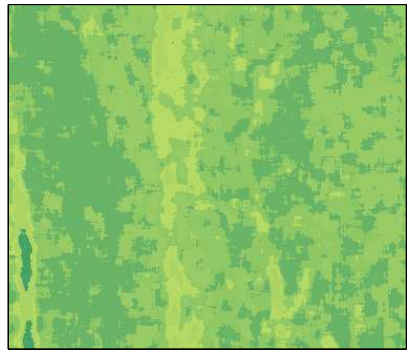

(b)(ii)

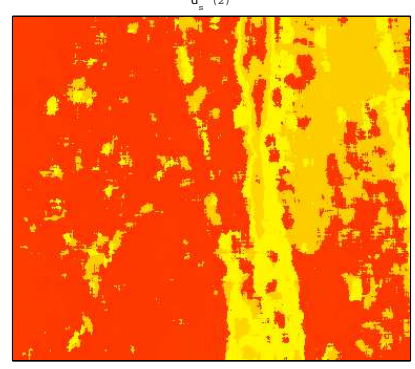

(c)(i)

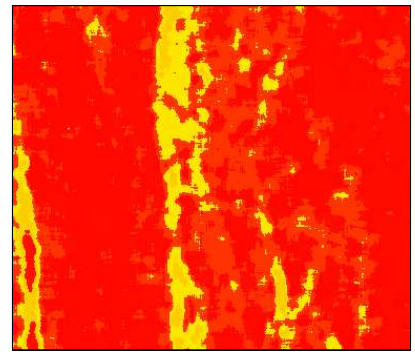

(c)(ii)

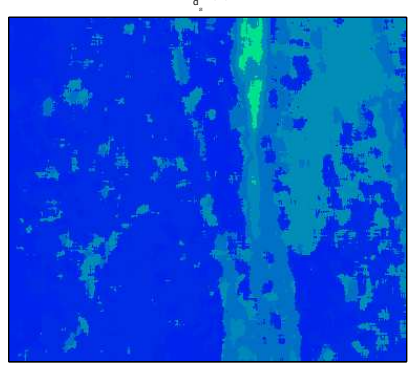

(d)(i)

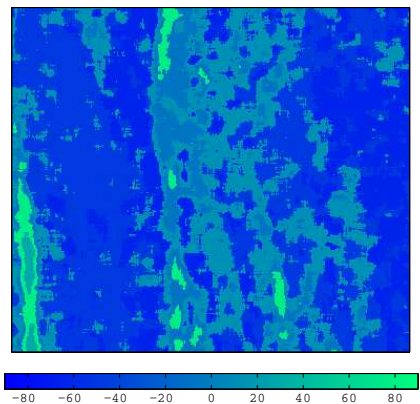

(d)(ii)

Fig. 9: ALOS L-band image I (i) and image II (ii) acquired over Chamonix, Mont Blanc, France: (a) classification, (b) $\tau_{m}(2)$ (ICA-C2), (c) $\alpha_{s}(2)$ (ICA-C2), (d) $\Phi_{\alpha_{s}}(2)$ (ICA-C2). (iii) Poincare sphere representation of the "locally" derived 2nd dominant components: (a) bare ground class (I/1), (b) wet snow class (I/2), (c) wet snow class (II/1), (d) dry snow class (II/2), (e) dry snow class (II/3).

[2] _ "Measurement of the target scattering matrix," Proc. IEEE, vol. 53, no. 8, pp. 936-946, 1965.

[3] S. R. Cloude and E. Pottier, "A review of target decomposition theorems in radar polarimetry," IEEE Trans. Geosci. Remote Sens., vol. 34, no. 2, pp. 498-518, 1996.

[4] D. Massonet and J.-C. Souyris, Imaging with Synthetic Aperture Radar, 1st ed. Boca Raton, FL, USA: CRC Press, Taylor and Francis Group, 2008.

[5] S. R. Cloude and E. Pottier, "An entropy based classification scheme for land applications of polarimetric sar," IEEE Trans. Geosci. Remote Sens., vol. 35, no. 1, pp. 68-78, 1997.

[6] R. Touzi, "Target scattering decomposition in terms of roll-invariant target properties," IEEE Trans. Geosci. Remote Sens., vol. 45, no. 1, pp. 73-84, 2007.

[7] P. Comon, "Independent component analysis: A new concept?" Signal Processing, vol. 36, pp. 287-314, 1994.

[8] I. T. Jolliffe, Principal Component Analysis, 2nd ed. Springer Series in Statistics, 2002.

[9] A. Papoulis, Probability, Random Variables and Stochastic Processes, 3rd ed. New York, NY, USA: McGraw-Hill, 1991.

[10] A. Hyvarinen and E. Oja, "Independent component analysis: Algorithms and applications," Neural Networks, vol. 13, no. 4-5, pp. 411-430, 2000.

[11] F. Totir, G. Vasile, L. Bombrun, and M. Gay, "Polsar images characterization through blind source separation techniques," in Proc. IGARSS, Hawaii, USA, 2010, pp. 4039-4042.

[12] C. Lopez-Martinez and X. Fabregas, "Polarimetric sar speckle noise model," IEEE Trans. Geosci. Remote Sens., vol. 41, no. 10, pp. 2232 2242, 2003.

[13] G. Vasile, J. P. Ovarlez, F. Pascal, and C. Tison, "Coherency matrix estimation of heterogeneous clutter in high-resolution polarimetric sar images," IEEE Trans. Geosci. Remote Sens., vol. 48, no. 4, pp. 18091826, 2010.
[14] S. Fiori, "Overview of independent component analysis technique with an application to synthetic aperture radar (sar) imagery processing," Neural Networks, vol. 16, no. 3-4, pp. 453-467, 2003.

[15] C. H. Chen and X. Zhan, "On the roles of independent component analysis in remote sensing," in Proc. of Progress in Electromagnetics Research Symposium, Cambridge, MA, USA, 2000.

[16] O. Tannous and D. Kasilingam, "Independent component analysis of polarimetric sar data for separating ground and vegetation components," in Proc. IGARSS, vol. IV, Cape Town, ZA, 2009, pp. 93-96.

[17] H. Yamada, R. Takizawa, Y. Yamaguchi, and R. Sato, "Polsar/pol-insar data analysis by using ica," in EUSAR 2010, Aachen, DE, 2010.

[18] P. Formont, F. Pascal, G. Vasile, J.-P. Ovarlez, and L. Ferro-Famil, "Statistical classification for heterogeneous polarimetric sar images," IEEE J. Sel. Topics Signal Process., vol. 5, no. 3, pp. 398-407, 2011.

[19] M. Novey and T. Adali, "On extending the complex fastica algorithm to noncircular sources," IEEE Trans. Signal Processing, vol. 56, no. 5, pp. 2148-2154, 2008.

[20] N. Besic, G. Vasile, J. Chanussot, and S. Stankovic, "Poincare sphere representation of independent scattering sources: application on distributed targets," in ESA SP-713 - POLinSAR 2013, Frascati, IT, 2013.

[21] R. Paladini, L. Ferro-Famil, E. Pottier, M. Mortorella, F. Berizzi, and E. D. Mese, "Lossless and sufficient orientation invariant decomposition of random reciprocal target," IEEE Trans. Geosci. Remote Sens., vol. 50, no. 9, pp. 3487-3501, 2012.

[22] P. Common and C. Jutten, Handbook of blind source separation, independent component analysis and applications. Oxford, UK: Academic Press, 2010

[23] M. S. Greco and F. Gini, "Statistical analysis of high-resolution sar ground clutter data," IEEE Trans. Geosci. Remote Sens., vol. 45, no. 3, pp. 566-575, 2007.

[24] N. Besic, G. Vasile, J. Chanussot, S. Stankovic, D. Boldo, and G. d'Urso, "Independent component analysis within polarimetric incoherent target 
TABLE III: RAMSES X-BAND POLSAR DATA OVER BRÉTIGNY, FRANCE: COMPARISON OF THE DERIVED TSVM, CLOUDE-POTTIER AND CPSV PARAMETERS

\begin{tabular}{|c|c|c|c|c|c|c|c|c|c|c|c|c|c|}
\hline \multirow{2}{*}{$\begin{array}{c}\text { Parameter } \\
\text { (rotation) }\end{array}$} & \multirow{2}{*}{ Method } & \multicolumn{3}{|c|}{ Class II } & \multicolumn{3}{|c|}{ Class IV } & \multicolumn{3}{|c|}{ Class VI } & \multicolumn{3}{|c|}{ Class VIII } \\
\hline & & $1 \mathrm{st}$ & 2nd & 3 rd & $1 \mathrm{st}$ & 2nd & 3rd & $1 \mathrm{st}$ & 2nd & 3rd & $1 \mathrm{st}$ & 2nd & 3 rd \\
\hline \multirow{2}{*}{$\tau_{m}\left[^{\circ}\right]\left(-20^{\circ}\right)$} & $\overline{\mathrm{PCA}}$ & 0.04 & 43.25 & 42.21 & -0.22 & 2.71 & -42.84 & -6.71 & 1.7 & -42.87 & -0.23 & -37.15 & 36.15 \\
\hline & ICA & -0.15 & -25.12 & 42.73 & -0.64 & 1.27 & 2.92 & -4.71 & 6.15 & -29.42 & -0.28 & -0.24 & 19.84 \\
\hline \multirow{2}{*}{$\tau_{m}\left[^{\circ}\right]\left(0^{\circ}\right)$} & PCA & 0.04 & 43.25 & 42.21 & -0.22 & 2.71 & -42.84 & -6.71 & 1.7 & -42.87 & -0.23 & -37.15 & 36.15 \\
\hline & ICA & -0.15 & -25.12 & 42.73 & -0.64 & 1.27 & 2.92 & -4.71 & 6.15 & -29.42 & -0.28 & -0.24 & 19.84 \\
\hline \multirow{2}{*}{$\tau_{m}\left[^{\circ}\right]\left(20^{\circ}\right)$} & PCA & 0.04 & 43.25 & 42.21 & -0.22 & 2.71 & -42.84 & -6.71 & 1.7 & -42.87 & -0.23 & -37.15 & 36.15 \\
\hline & ICA & -0.15 & -25.12 & 42.73 & -0.64 & 1.27 & 2.92 & -4.71 & 6.15 & -29.42 & -0.28 & -0.24 & 19.84 \\
\hline \multirow{2}{*}{$\Phi_{\alpha_{s}}\left[^{\circ}\right]\left(-20^{\circ}\right)$} & PCA & -34 & 20.19 & -24.89 & -38.39 & 38.36 & -84.16 & 6.02 & -5.72 & 69.01 & -51.25 & -18.64 & 68.86 \\
\hline & ICA & 9.6 & -11.65 & -34.12 & -38.92 & 39.32 & -34.23 & -1.83 & 26.25 & -74.18 & -27.42 & 2.56 & 77.92 \\
\hline \multirow{2}{*}{$\Phi_{\alpha_{s}}\left[^{\circ}\right]\left(0^{\circ}\right)$} & PCA & -34 & 20.19 & -24.89 & -38.39 & 38.36 & -84.16 & 6.02 & -5.72 & 69.01 & -51.25 & -18.64 & 68.86 \\
\hline & ICA & 9.6 & -11.65 & -34.12 & -38.92 & 39.32 & -34.23 & -1.83 & 26.25 & -74.18 & -27.42 & 2.56 & 77.92 \\
\hline \multirow{2}{*}{$\Phi_{\alpha_{s}}\left[^{\circ}\right]\left(20^{\circ}\right)$} & PCA & -34 & 20.19 & -24.89 & -38.39 & 38.36 & -84.16 & 6.02 & -5.72 & 69.01 & -51.25 & -18.64 & 68.86 \\
\hline & ICA & 9.6 & -11.65 & -34.12 & -38.92 & 39.32 & -34.23 & -1.83 & 26.25 & -74.18 & -27.42 & 2.56 & 77.92 \\
\hline \multirow{2}{*}{$\alpha_{s}\left[^{\circ}\right]\left(-20^{\circ}\right)$} & PCA & 1.35 & 78.48 & 78.02 & 39.9 & 49.95 & 64.49 & 68.3 & 20.85 & 73.19 & 0.5 & 89.21 & 87.9 \\
\hline & ICA & 5.03 & 71.48 & 18.74 & 41.7 & 47.1 & 84.59 & 62.19 & 58.22 & 33.23 & 0.53 & 39.91 & 58.49 \\
\hline \multirow{2}{*}{$\alpha_{s}\left[^{\circ}\right]\left(0^{\circ}\right)$} & PCA & 1.35 & 78.48 & 78.02 & 39.9 & 49.95 & 64.49 & 68.3 & 20.85 & 73.19 & 0.5 & 89.21 & 87.9 \\
\hline & ICA & 5.03 & 71.48 & 18.74 & 41.7 & 47.1 & 84.59 & 62.19 & 58.22 & 33.23 & 0.53 & 39.91 & 58.49 \\
\hline \multirow{2}{*}{$\alpha_{s}\left[^{\circ}\right]\left(20^{\circ}\right)$} & $\mathrm{PCA}$ & 1.35 & 78.48 & 78.02 & 39.9 & 49.95 & 64.49 & 68.3 & 20.85 & 73.19 & 0.5 & 89.21 & 87.9 \\
\hline & ICA & 5.03 & 71.48 & 18.74 & 41.7 & 47.1 & 84.59 & 62.19 & 58.22 & 33.23 & 0.53 & 39.91 & 58.49 \\
\hline \multirow{2}{*}{$\Upsilon_{\mathrm{TSVM}}\left[^{0}\right]$} & PCA & 14 & 17.45 & 16.28 & 12.9 & 12.91 & 43.54 & 24.01 & 23.93 & 5.25 & 35.31 & 17.84 & 39.71 \\
\hline & ICA & 20.1 & 19.59 & 13.97 & 12.77 & 12.67 & 31.06 & 22.04 & 15.94 & 3.96 & 29.35 & 23.14 & 3.02 \\
\hline \multirow{2}{*}{$\mathrm{Hel}_{\mathrm{TSVM}}$} & PCA & 0 & 0.37 & -0.37 & 0.01 & 0.07 & -0.08 & 0.16 & 0.04 & -0.2 & 0 & 0.03 & -0.03 \\
\hline & ICA & 0 & 0.45 & -0.5 & 0.02 & 0.03 & 0.02 & 0.14 & 0.17 & 0.21 & 0 & 0.01 & 0.12 \\
\hline \multirow{2}{*}{$\Upsilon_{c}\left[^{\circ}\right]$} & $\overline{\mathrm{PCA}}$ & 31.02 & -17.25 & -61.03 & 32.1 & -12.87 & 1.19 & 20.98 & -23.97 & -4.84 & 50.37 & 27.16 & -39.72 \\
\hline & ICA & -20.09 & 25.61 & -45.8 & 32.23 & -12.66 & -31.06 & 22.96 & -15.84 & 43.55 & 52.13 & 21.86 & -47.63 \\
\hline \multirow{2}{*}{$\mathrm{Hel}_{c}$} & PCA & 0 & 0.37 & -0.37 & 0.01 & 0.07 & -0.08 & 0.16 & 0.04 & -0.2 & 0 & 0.03 & -0.03 \\
\hline & ICA & 0 & 0.45 & -0.5 & 0.02 & 0.03 & 0.02 & 0.14 & 0.17 & 0.21 & 0 & 0.01 & 0.12 \\
\hline \multirow{2}{*}{$\alpha_{c}\left[^{\circ}\right]$} & PCA & 1.35 & 89.3 & 88.84 & 39.9 & 50.16 & 88.14 & 68.93 & 21.11 & 88.77 & 0.67 & 89.78 & 89.36 \\
\hline & ICA & 5.04 & 78.28 & 85.69 & 41.72 & 47.15 & 84.62 & 62.6 & 59.03 & 64.36 & 0.77 & 39.91 & 66.28 \\
\hline \multirow{2}{*}{$\alpha_{p}\left[^{\circ}\right]$} & $\overline{\mathrm{PCA}}$ & 1.35 & 89.3 & 88.84 & 39.9 & 50.16 & 88.14 & 68.93 & 21.11 & 88.77 & 0.67 & 89.78 & 89.36 \\
\hline & ICA & 5.04 & 78.28 & 85.69 & 41.72 & 47.15 & 84.62 & 62.6 & 59.03 & 64.36 & 0.77 & 39.91 & 66.28 \\
\hline \multirow{2}{*}{$\beta_{p}\left[^{\circ}\right]$} & PCA & 76.06 & 20.19 & 69.8 & 1.12 & 4.74 & 86.79 & 7.66 & 10.88 & 81.95 & 70.71 & 0.81 & 89.19 \\
\hline & ICA & 6.61 & 22.51 & 66.92 & 1.41 & 3.58 & 88.91 & 7.25 & 8.99 & 53.49 & 57.56 & 2.4 & 76.58 \\
\hline \multirow{2}{*}{$\gamma_{p}\left[^{\circ}\right]$} & PCA & 33.4 & -127.96 & -150.67 & 59.79 & -92.94 & 94.85 & 36.97 & -132.16 & 71.22 & 111.26 & 81.9 & -68.88 \\
\hline & ICA & 158.49 & 49.66 & -88.08 & 91.17 & -109.95 & -34.24 & 44.57 & -105.09 & 89.8 & 111.33 & 11.32 & -101.44 \\
\hline & PCA & -134.2 & 17.55 & 174.86 & -141.61 & 38.34 & 49.92 & 173.41 & -6.87 & 25.73 & 19.77 & -161.36 & -132.05 \\
\hline$o_{p}[]$ & ICA & 9.98 & -172.32 & -132.54 & -141.08 & 39.27 & -9.49 & -178.62 & 25.94 & -106.17 & 9.6 & 177.42 & -83.09 \\
\hline
\end{tabular}

decomposition," in Proc. IGARSS, Melbourne, AUS, 2013, pp. 41584161.

[25] A. Hyvarinen, "Fast and robust fixed-point algorithms for independent component analysis," IEEE Trans. on Neural Netw., vol. 10, no. 3, p. 626634, 1999

[26] E. Bingham and A. Hyvrinen, "A fast fixed-point algorithm for independent component analysis of complex valued signals," Int. J. Neural Syst., vol. 10, no. 1, p. 18, 2000.

[27] R. A. Horn and C. R. Johnson, Matrix Analysis. Oxford, UK: Cambridge University Press, 1990, ch. Norms for Vectors and Matrices.

[28] E. M. Kennaugh, "Polarization properterties of radar reflection," Ph.D. dissertation, Dept. Elect. Eng., Ohio State Univ., Columbus, OH, USA, 1952.

[29] R. Touzi and F. Charbonneau, "Characterization of target symmetric scattering using polarimetric sars," IEEE Trans. Geosci. Remote Sens., vol. 40, no. 11, pp. 2507-2516, 2002.

[30] S. R. Cloude, "The characterization of polarization effect in em scattering," Ph.D. dissertation, University of Birmingham, Birmingham, UK, 1986.

[31] J. S. Lee, M. R. Grunes, T. L. Ainsworth, D. Li-Jen, D. L. Schuler, and S. R. Cloude, "Unsupervised classification using polarimetric decomposition and the complex wishart classifier," IEEE Trans. Geosci. Remote Sens., vol. 37, no. 5, pp. 2249-2258, 1999.

[32] J. S. Lee, M. R. Grunes, E. Pottier, and L. Ferro-Famil, "Unsupervised terrain classification preserving polarimetric scattering characteristics,' IEEE Trans. Geosci. Remote Sens., vol. 42, no. 4, pp. 722-731, 2004.

[33] K. Yao, "A representation theorem and its applications to sphericallyinvariant random processes," IEEE Trans. Geosci. Remote Sens., vol. IT-19, no. 5, pp. 600-608, 1973.

[34] F. Pascal, Y. Chitour, J. P. Ovarlez, P. Forsterand, and P. Larzabal, "Covariance structure maximum likelihood estimates in compound gaussian noise: Existence and algorithm analysis," IEEE Trans. Signal Process., vol. 56, no. 1, pp. 34-38, 2008.
[35] R. C. Gonzales and R. E. Woods, Digital Image Processing. Reading, Massachusetts, USA: Addison-Wesley, 1993.

[36] R. Paladini, L. Ferro-Famil, E. Pottier, M. Mortorella, F. Berizzi, and E. D. Mese, "Point target classification via fast lossless and sufficient $\omega-$ $\psi-\phi$ invariant decomposition of high-resolution and fully polarimetric sar/isar data," Proc. IEEE, vol. 101, no. 3, pp. 798-830, 2013.

[37] R. Paladini, L. Ferro-Famil, E. Pottier, M. Mortorella, and F. Berizzi, "Lossless and sufficient orientation invariant decomposition of random target," in ESA SP-695 - POLinSAR 2013, Frascati, IT, 2011.

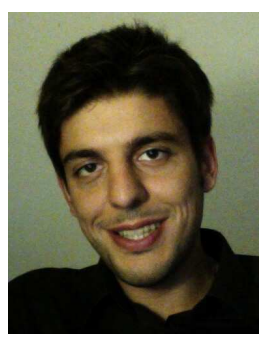

Nikola Besic (S'10) was born in Podgorica, Montenegro, in November 1987. He received the B.Sc. degree in electrical engineering in 2009 from the University of Montenegro and the M.Sc. degree in optics and RF engineering in 2011 from the Grenoble Institute of Technology (Phelma/Grenoble INP). Currently, as a French Government fellow, with the Grenoble-Image-sPeech-Signal-Automatics Laboratory (GIPSA-lab) in Grenoble, he is jointly pursuing the Doctorate degree in signal and image processing, from the University of Grenoble, and the Ph.D. degree in technical sciences, from the University of Montenegro.

His current research is mostly related to radar and optical remote sensing, with a particular emphasis on the radar polarimetry and the remote sensing of cryosphere. His research interests also include other signal and image processing techniques, tightly bound to physics of electromagnetic and mechanical waves 


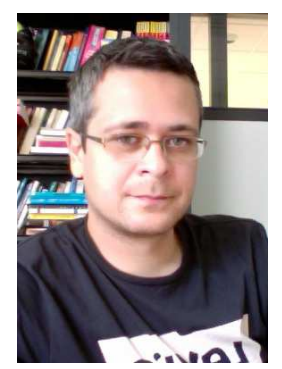

Gabriel Vasile (S'06-M'07) received the M.Eng. degree in electrical engineering and computer science and the M.S. degree in image, shapes, and artificial intelligence from the POLITEHNICA University, Bucharest, Romania, in 2003 and 2004, respectively, and the Ph.D. degree in signal and image processing from the Savoie University, Annecy, France, in 2007. From 2007 to 2008, he was a Postdoctoral Fellow with the French Space Agency (CNES) and was with the French Aerospace Laboratory (ONERA), Palaiseau, France. In 2008, he joined the French National Council for Scientific Research (CNRS), where he is currently a Research Scientist and a member of the Grenoble Image Speech Signal Automatics Laboratory, Grenoble, France.

$\mathrm{He}$ is author of more than 100 papers published in international journals and conference proceedings. In 2008, he received the "Bourse de la Vocation Prix des Neiges" to recognize his work in radar remote sensing for the benefit of the Savoie and the Haute Savoie counties, France.

His current research interests include signal and image processing, radar and acoustics remote sensing, polarimetry, interferometry, and tomography.

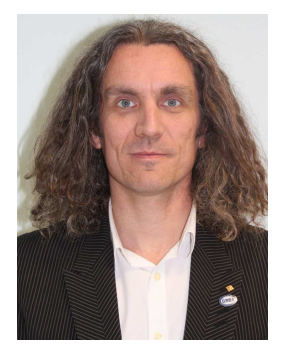

Jocelyn Chanussot (M'04-SM'04-F'12) received the M.Sc. degree in electrical engineering from the Grenoble Institute of Technology (Grenoble INP), Grenoble, France, in 1995, and the Ph.D. degree from Savoie University, Annecy, France, in 1998. In 1999, he was with the Geography Imagery Perception Laboratory for the Delegation Generale de l'Armement (DGA - French National Defense Department). Since 1999, he has been with Grenoble INP, where he was an Assistant Professor from 1999 to 2005, an Associate Professor from 2005 to 2007, and is currently a Professor of signal and image processing. $\mathrm{He}$ is conducting his research at the Grenoble Images Speech Signals and Automatics Laboratory (GIPSA-Lab). His research interests include image analysis, multicomponent image processing, nonlinear filtering, and data fusion in remote sensing. He is a member of the Institut Universitaire de France (2012-2017). Since 2013, he is an Adjunct Professor of the University of Iceland.

Dr. Chanussot is the founding President of IEEE Geoscience and Remote Sensing French chapter (2007-2010) which received the 2010 IEEE GRSS Chapter Excellence Award. He was the co-recipient of the NORSIG 2006 Best Student Paper Award, the IEEE GRSS 2011 Symposium Best Paper Award, the IEEE GRSS 2012 Transactions Prize Paper Award and the IEEE GRSS 2013 Highest Impact Paper Award. He was a member of the IEEE Geoscience and Remote Sensing Society AdCom (2009-2010), in charge of membership development. He was the General Chair of the first IEEE GRSS Workshop on Hyperspectral Image and Signal Processing, Evolution in Remote sensing (WHISPERS). He was the Chair (2009-2011) and Cochair of the GRS Data Fusion Technical Committee (2005-2008). He was a member of the Machine Learning for Signal Processing Technical Committee of the IEEE Signal Processing Society (2006-2008) and the Program Chair of the IEEE International Workshop on Machine Learning for Signal Processing, (2009). He was an Associate Editor for the IEEE Geoscience and Remote Sensing Letters (2005-2007) and for Pattern Recognition (2006-2008). Since 2007, he is an Associate Editor for the IEEE Transactions on Geoscience and Remote Sensing. Since 2011, he is the Editor-in-Chief of the IEEE Journal of Selected Topics in Applied Earth Observations and Remote Sensing. In 2013, he was a Guest Editor for the Proceedings of the IEEE and in 2014 a Guest Editor for the IEEE Signal Processing Magazine. He is a Fellow of the IEEE and a member of the Institut Universitaire de France (2012-2017).

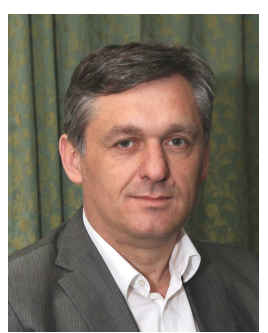

Srdjan Stankovic was born 1964 in Montenegro. He received the B.S. (Hons.) degree from the University of Montenegro, in 1988, the M.S. degree from the University of Zagreb, Croatia, in 1991, and the Ph.D. degree from the University of Montenegro in 1993, all in Electrical Engineering.

From 1988 to 1992 , he worked in the Aluminum Plant of Podgorica as a Research Assistant. In 1992 he joined the Faculty of Electrical Engineering, University of Montenegro, where he is currently a Full Professor. In the period 2007-2013 he served as Dean of the Faculty of Electrical Engineering, University of Montenegro. His interests are in signal processing, multimedia systems, and digital electronics. $\mathrm{He}$ is a member of the Board of Directors in Montenegrin Broadcasting Company since 2004. He was the President of the Board of Directors in Montenegrin Broadcasting Company (2005-2006). In 1998 he spent a period of time with the Department of Informatics at the Aristotle University in Thessaloniki, supported by Greek IKY foundation. In the 1999-2000, he was on leave at the Darmstadt University of Technology, with the Signal Theory Group, supported by the Alexander von Humboldt Foundation. In 2002, he spent three months at the Department of Computer Science, the University of Applied Sciences Bonn-Rhein-Sieg, as an Alexander von Humboldt Fellow. From 2004 to 2006, he stayed several times with the E3I2 Laboratory, ENSIETA, Brest, France. From 2007 to 2010 he visited (one month research stay) Centre for digital signal processing research at King's College London, Laboratory of mathematical methods in image processing, at Moscow State University Lomonosov, as well as GIPSA Laboratory at INPG Grenoble. He spent academic 2012/2013 with the Center for Advanced Communications at the Villanova University, PA.

He has published a book "Multimedia Signals and Systems" by Springer and several textbooks on electronics devices (in Montenegrin). He published about 80 journal papers. In 2010, he was the Lead Guest Editor of the EURASIP Journal on Advances in Signal Processing for the special issue: Time-frequency analysis and its applications to multimedia signals, as well as the Guest Editor of the Signal Processing for special issue: Fourier related transforms. He was the Lead Guest Editor of the IET Signal Processing for the Special issue: Compressive Sensing and Robust Transforms, published in 2014.

From 2005 to 2009 Dr. Stankovic was serving as an Associate Editor of the IEEE Transactions on Image Processing. He is a Senior member of the IEEE. In 2011 he was awarded by the Ministry of Science in Montenegro as the Leader of the Best Scientific Project in Montenegro. 